\title{
Determination of Plasma Temperature by a Semi-Empirical Method
}

\author{
F. O. Borges, G. H. Cavalcanti, and A. G. Trigueiros \\ Laboratório de Plasma e Espectroscopia, Instituto de Física, Universidade Federal Fluminense, UFF \\ Campus da Praia Vermelha-Gragoatá, 24210-340 Niterói, Rio de Janeiro, Brazil
}

Received on 26 January, 2004; revised version received on 28 April, 2004

\begin{abstract}
Doppler or Stark line broadening effects are generally used to determinate plasma temperature. These methods are difficult to apply to spectra of highly ionized atoms due to the short wavelengths involved. It is not at all easy to achieve sufficient wavelength resolution in this spectral range. In this case, a spectroscopic technique based on the relative intensities of lines must be used to measure the electron temperature in a plasma. However the relation of the measure of relative line intensity and the plasma electron temperature is complex and a number of issues must be examined for the diagnostic. In simple cases, only a two levels system need be considered. Here we introduce a semi-empirical method to determine the plasma temperature that takes into account multiple levels structure. In the theoretical model we consider a local thermodynamic equilibrium(LTE). The greatest difficult in the determination of plasma temperature using a multiple levels approach is overcome by calculating the transition probabilities in terms of the oscillator strength parameters. To check the method we calculated the oscillator strengths for the $\mathrm{Cu}$ I using a multi-configurational Hartree-Fock relativistic (HFR) approach. The electrostatic parameters were optimized by a least-squares procedure, in order to obtain the best fitting to the experimental energy levels. This method produces $g f$ - values that are in better agreement with their experimental values than the produced by the ab initio calculation. The temperature obtained was $5739.3 \mathrm{~K}$, what is compatible with direct measurements made for cupper DC discharge.
\end{abstract}

\section{Determination of plasma tempera- ture}

The plasma temperature may be estimated through the relative emission intensities of spectral lines. From the theory of atomic structure and spectra [1], the relative intensities of the spectral lines, considering a Boltzmann distribution, are given by the equation

$$
\ln \left(\frac{I \lambda^{3}}{g f}\right)=-\frac{E_{u}}{k_{B} T}+\text { constant }=-a E_{u}+b,
$$

where $I, \lambda$ and $f$ are the relative intensity, wavelength, and oscillator strength respectively. $E_{u}$ is the energy of the upper level and $g$ is the statistic weight of the lower level; $k_{B}$ is the Boltzmann constant, $T$ is the temperature and $b$ a constant parameter for all ion lines considered. In a few cases, $g, f$ and $E_{u}$ can be obtained from the handbook of the spectroscopic constants, chemistry and physics.

The equation(1) gives the plasma temperature in the case of LTE approximation. To increase the precision a large number of spectral lines must be used to determine plasma temperature. For a given spectrum, a plot of the logarithmic term in the right hand of equation(1) versus $E_{u}$ yields a straight line whose slope is equal to $1 / k_{B} T$. Thus we can obtain the plasma temperature from the slope $a$. Using

$$
T=\frac{1}{k_{B} a} .
$$

We apply the above mentioned method to some copper lines which spectroscopic constants are listed in Table 2.
The major difficult here, is to obtain the oscillator strengths for the observed lines which are absent in the literature. Therefore to obtain the plasma temperature and overcome this diffcult it is necessary to obtain this data in a semi-empirical way.

\section{Semi-empirical $g f$ calculation}

The oscillator strength $f\left(\gamma \gamma^{\prime}\right)$ is a physical quantity related to line intensity $I$ and transition probability $W\left(\gamma \gamma^{\prime}\right)$. According to Sobelman [2] it may be writen as:

$$
W\left(\gamma \gamma^{\prime}\right)=\frac{2 \omega^{2} e^{2}}{m c^{3}}\left|f\left(\gamma \gamma^{\prime}\right)\right|,
$$

where $m$ is the electron mass, $e$ is the electron charge, $\gamma$ is the initial quantum state, $\omega=\left(E(\gamma)-E\left(\gamma^{\prime}\right)\right) / \hbar, E(\gamma)$ is the energy of initial state, $g=(2 J+1)$ is the number of degenerate quantum states with angular momentum $J$ (in the formula for the initial state). Quantities with primes refer to the final state. The intensity proportionality may be expressed as:

$$
I \propto g^{\prime} W\left(\gamma \gamma^{\prime}\right) \propto g^{\prime}\left|f\left(\gamma \gamma^{\prime}\right)\right|=g^{\prime} f
$$

In the equation above, the weighted oscillator strength, $g f$, is given by Cowan [3] as:

$$
g^{\prime} f=\frac{8 \pi^{2} m c a_{0}^{2} \sigma}{3 h} S
$$


where $\sigma=\left|E(\gamma)-E\left(\gamma^{\prime}\right)\right| / h c, h$ is the Planck's constant, $\mathrm{c}$ is the light velocity, and $a_{0}$ is the Bohr radius, and the electric dipole line strength is defined by:

$$
\mathbf{S}=\left|<\gamma J\left\|\mathbf{P}^{1}\right\| \gamma^{\prime} J^{\prime}>\right|^{2} .
$$

This quantity is a measure of the total strength of the spectral line, including all the possible transitions between

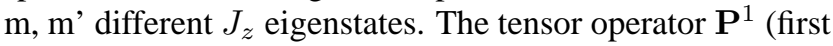
order) in the reduced matrix element is the classical dipole moment for the atom in units of $e a_{0}$.

To obtain $g f$ we need to calculate $\mathbf{S}$ first or its square root through:

$$
\mathbf{S}_{\gamma \gamma^{\prime}}^{1 / 2}=<\gamma J\left\|\mathbf{P}^{1}\right\| \gamma^{\prime} J^{\prime}>.
$$

In a multi-configurational calculation it is necessary to expand the wavefunction $\mid \gamma J>$ in terms of single configuration wavefunctions, $\mid \beta J>$, for both upper and lower levels. The wavefunctions may be expressed as:

$$
\left|\gamma J>=\sum_{\beta} y_{\beta J}^{\gamma}\right| \beta J>.
$$

Therefore the multiconfigurational expression for $S_{\gamma \gamma^{\prime}}^{1 / 2}$ in this case is

$$
\mathbf{S}_{\gamma \gamma^{\prime}}^{1 / 2}=\sum_{\beta} \sum_{\beta^{\prime}} y_{\beta J}^{\gamma}<\beta J\left\|\mathbf{P}^{1}\right\| \beta^{\prime} J^{\prime}>y_{\beta^{\prime} J^{\prime}}^{\gamma^{\prime}}
$$

The probability per unit time of an atom in a specific state $\mid \gamma J>$ to make a spontaneous transition to any state with lower energy is

$$
P(\gamma J)=\sum A\left(\gamma J, \gamma^{\prime} J^{\prime}\right)
$$

where $A\left(\gamma J, \gamma^{\prime} J^{\prime}\right)$ is the Einstein spontaneous emission transition probability rate for a transition from the $\mid \gamma J>$ to the $\mid \gamma^{\prime} J^{\prime}>$ state. The sum is over all $\mid \gamma^{\prime} J^{\prime}>$ states with $E\left(\gamma^{\prime} J^{\prime}\right)<E(\gamma J)$.

According to Cowan [3] the Einstein probability rate is related to $g f$ through the following relation:

$$
g A=\frac{8 \pi^{2} e^{2} \sigma^{2}}{m c} g^{\prime} f=0.6670 \times 10^{16} \frac{g^{\prime} f}{\lambda^{2}} .
$$

In order to obtain better values for oscillator strengths, we calculated the reduced matrix elements $\mathbf{P}^{1}$ by using optimized values of energy parameters which were adjusted from a least-squares procedure. In the adjustment, the code tries to fit the energy values to experimental ones by varying the electrostatic parameters. After, the improved wavenumbers are used in eq. (4) and $\mathrm{y}_{\beta J}^{\gamma}$ and $\mathrm{y}_{\beta J^{\prime}}^{\gamma^{\prime}}$ coefficients are used in eq. (8). The theoretical predictions for the energy levels of the configurations were obtained by diagonalizing of the energy matrices with appropriate option Hartree-Fock relativistic (HFR) using the computer code developed by Cowan [3].

The energy level values were determined from the observed wavelengths by an interactive optimization procedure using the program ELCALC(Energy Level Calculations), where the individual wavelengths are weighted according to their uncertainties [4]. The energy levels adjusted by this method were used to optimize the electrostatic parameters by a least-squares procedure, and finally these optimized parameters were used to calculate the $g f$-values. This method produces $g f$-values that are in better agreement with the line intensity observed, compared with $g f$ exclusively theoretical.

\section{Results and discussion}

We have calculated the theoretical Hartree-Fock values for atomic parameters of some $\mathrm{Cu}$ I configurations. The picture we used to perform the theoretical calculations: for even-parity configurations, the $3 d^{10} 4 s, 3 d^{9} 4 s^{2}, 3 d^{10} 4 d$, and $3 d^{9} 4 s 5 s$ are involved and for odd-parity configurations, the $3 d^{10} 4 p$ and $3 d^{9} 4 s 4 p$ are studied. Due to the high configuration interaction value, we have also included the configurations $3 d^{9} 4 p^{2}$ and $3 d^{9} 4 p 5 p$ to improve the even-parity estimatives and configuration $3 d^{10} 5 p$ into the theoretical calculation of the odd-parity states. Table 1 shows only the energy parameters for the configurations that are in focus of attention where appear some values for the configuration integral which are relevant in the present case. The Fig. 1 shows an illustration of a semi-empirical results for some $\mathrm{Cu} \mathrm{I}$ lines, where we present a plot of oscillator strengths versus wavelengths. The figure represents the spectral intensity distribution for copper lines related to the array transitions studied here.

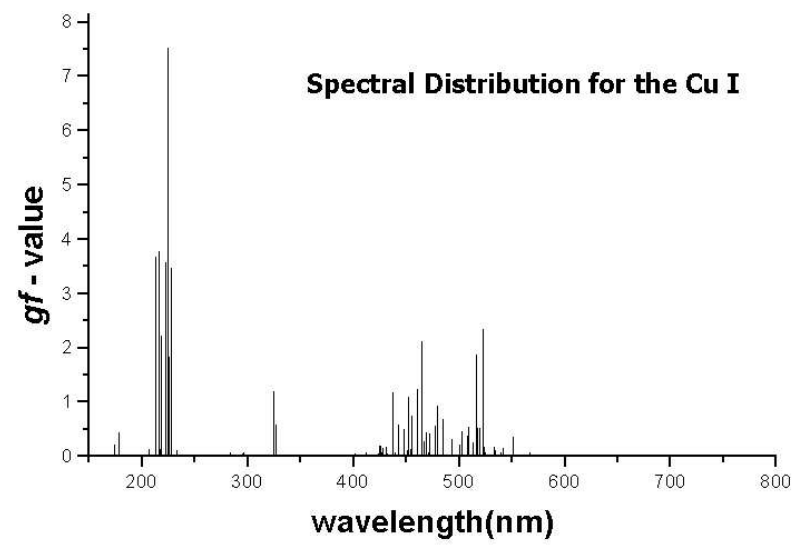

Figure 1. Semi-empirical spectrum for the transition of the $\mathrm{Cu} \mathrm{I}$.

Baoming and Hongzhi [5] have studied experimentally some $\mathrm{Cu}$ I transitions that are used in this work. Measurement of relative intensities of cupper lines are listed in this work. The atomic parameters used to perform the coefficients calculation of equation (1) are shown in Table 2, where is showed the experimental transition probabilities and semiempirical transition probabilities calculated here. The Fig. 2 shows the linear Boltzmann plot of equation (1) using the results of Baoming et al., that gives a plasma temperature of $5946.9 \mathrm{~K}$. The Fig. 3 shows a similar result $(\mathrm{T}=5739.3$ $\mathrm{K})$ to plasma temperature using the semi-empirical atomic parameters determined here. 


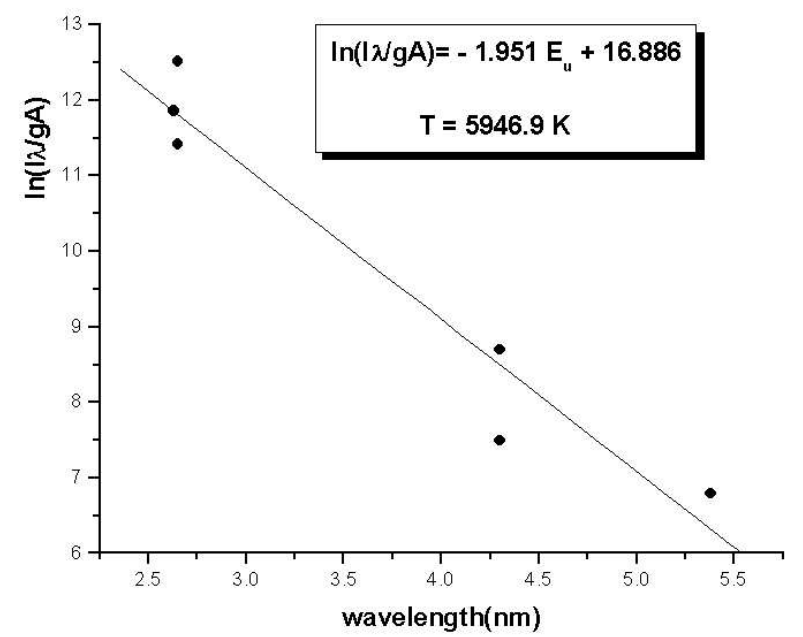

Figure 2. Linear plot of equation (1) using Baoming and Hongzhi [5] results for atomic parameters of the $\mathrm{Cu}$ I.

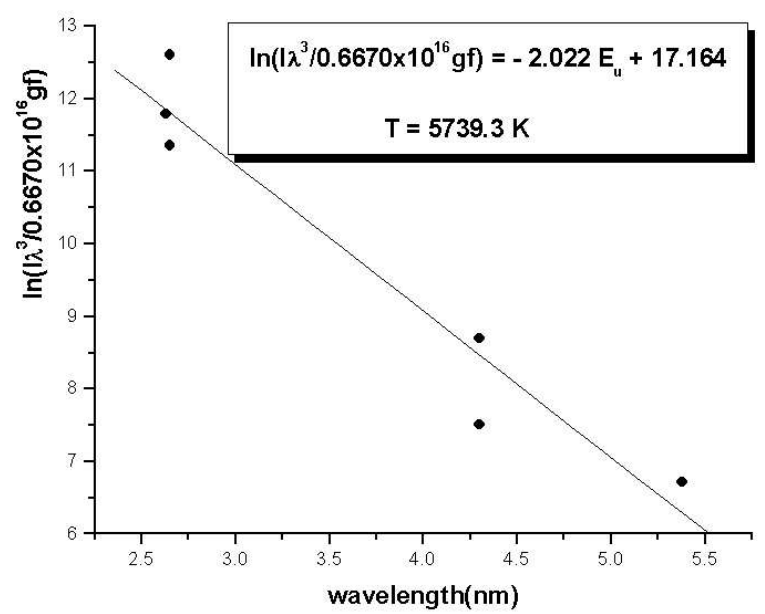

Figure 3. Boltzmann plot of equation (1) using semi-empirical $g f$ values calculated in this work for $\mathrm{Cu}$ I spectrum.

\section{Conclusions}

We hope that the semi-empirical method described in this work can be successfully applied to calculate electron temperature in plasmas. The semi-empirical oscillator strengths calculated by the fitting between theoretical and experimental energy levels produces very good values. This semi-empirical method produces a very good linear relationship between $\ln \left(I \lambda^{3} / g f\right)$ and $E_{u}$. Its applicability goes beyond the visible range and may be used in vacuum ultra violet range too. We have made a study of some $\mathrm{Cu}$ I lines and determined the plasma temperature of a DC plasma discharge that are in acordance with expected. Also, our result is in a very good agreement with the result of Baoming and Hongzhi. The semi-empirical method of measuring temperature gave a difference of $3.5 \%$.

We present here the oscillator strengths for some known electric dipole transitions in $\mathrm{Cu}$ I. The present work is part of an ongoing program, whose goal is to obtain weighted oscillator strength, $g f$, and lifetimes for elements of astrophysical importance. The ions S VII [7], S IX and S X [8], have been concluded.

\section{Acknowledgements}

This work was financially supported by the Conselho Nacional de Desenvolvimento Científico e Tecnológico, CNPq (Brazil), Coordenação de Aperfeiçoamento de Pessoal de Nível Superior, CAPES (Brazil), and by Fundação de Amparo à Pesquisa do Estado do Rio de Janeiro, FAPERJ (Brazil).

\section{References}

[1] H. R. Griem, Plasma Spectroscopy, McGraw-Hill: New York (1964).

[2] I. Sobelman, Atomic Spectra and Radiative Transitions. Springer: Berlin (1979).

[3] R. D. Cowan, The Theory of Atomic Structure and Spectra Berkeley, Univ California Press (1981).

[4] L. J. Radziemski and V. Kaufman, J. Opt. Soc. Am. 59, 424 (1969).

[5] Baoming Li and Hongzhi Li, 19th International Symposium of Ballistics, 7-11 May 2001, Interlaken, Switzerland.

[6] C. H. Corliss and W. R. Bozman, Experimental Transition Probabilities for Spectral Lines of Seventy Elementes, Washington: National Bureau of Standards Monograph S3, U. S. Government Printing Office (1962).

[7] F. O. Borges, G. H. Cavalcanti, A. G. Trigueiros, and C. Jupén, J. Quant. Spectrosc. Radiat. Transfer 83, 751 (2004).

[8] F. O. Borges, G. H. Cavalcanti, and A. G. Trigueiros, J. Quant. Spectrosc. Radiat. Transfer 78, 119 (2003). 
Table 1: Atomic energy parameters

\begin{tabular}{|c|c|c|c|c|}
\hline Configuration & Parameter & $\begin{array}{c}\mathrm{HF} \\
1000 \mathrm{~cm}^{-1}\end{array}$ & $\begin{array}{c}\text { Adjusted } \\
1000 \mathrm{~cm}^{-1}\end{array}$ & Adjust/HF \\
\hline $3 \mathrm{~d}^{10} 4 \mathrm{~s}$ & $E_{a v}$ & 0.000 & 0.000 & - \\
\hline \multirow[t]{2}{*}{$3 d^{9} 4 s^{2}$} & $E_{a v}$ & 11.580 & 12.209 & 1.054 \\
\hline & $\zeta_{3 d}$ & 0.810 & 0.817 & 1.009 \\
\hline \multirow[t]{2}{*}{$3 d^{10} 4 d$} & $E_{a v}$ & 45.590 & 49.942 & 1.095 \\
\hline & $\zeta_{4 d}$ & 0.001 & fix & - \\
\hline \multirow{5}{*}{$3 \mathrm{~d}^{9} 4 \mathrm{~s} 5 \mathrm{~s}$} & $E_{a v}$ & 46.167 & 64.551 & 1.398 \\
\hline & $\zeta_{3 d}$ & 0.814 & 0.826 & 1.015 \\
\hline & $G^{2}(3 d 4 s)$ & 9.866 & 7.828 & 0.793 \\
\hline & $G^{2}(3 d 5 s)$ & 0.711 & 0.676 & 0.951 \\
\hline & $G^{0}(4 s 5 s)$ & 1.665 & 0.934 & 0.561 \\
\hline \multirow[t]{2}{*}{$3 d^{10} 4 p$} & $E_{a v}$ & 27.830 & 31.752 & 1.141 \\
\hline & $\zeta_{4 p}$ & 0.129 & 0.183 & 1.419 \\
\hline \multirow[t]{8}{*}{$3 d^{9} 4 s 4 p$} & $E_{a v}$ & 28.528 & 46.553 & 1.632 \\
\hline & $\zeta_{3 d}$ & 0.813 & 0.663 & 0.815 \\
\hline & $\zeta_{4 p}$ & 0.248 & 0.203 & 0.819 \\
\hline & $F^{2}(3 d 4 p)$ & 9.289 & 9.185 & 0.989 \\
\hline & $G^{2}(3 d 4 s)$ & 8.749 & 8.109 & 0.927 \\
\hline & $G^{1}(3 d 4 p)$ & 3.348 & 3.103 & 0.927 \\
\hline & $G^{3}(3 d 4 p)$ & 2.619 & 2.428 & 0.927 \\
\hline & $G^{1}(4 s 4 p)$ & 33.176 & 20.699 & 0.624 \\
\hline \multicolumn{5}{|c|}{ Integral of Configuration Interaction } \\
\hline $3 d^{10} 4 s-3 d^{9} 4 s^{2}$ & $R_{d}^{2}(3 d 3 d, 3 d 4 s)$ & -3.744 & fix & - \\
\hline $3 d^{10} 4 s-3 d^{9} 4 s 5 s$ & $R_{d}^{2}(3 d 3 d, 3 d 5 s)$ & -0.941 & fix & - \\
\hline $3 d^{10} 4 s-3 d^{9} 4 p^{2}$ & $R_{d}^{1}(3 d 4 s, 4 p 4 p)$ & -11.396 & fix & - \\
\hline \multirow{2}{*}{$3 d^{10} 4 s-3 d^{9} 4 p 5 p$} & $R_{d}^{1}(3 d 4 s, 4 p 5 p)$ & -3.344 & fix & - \\
\hline & $R_{e}^{1}(3 d 4 s, 4 p 5 p)$ & -4.126 & fix & - \\
\hline $3 d^{9} 4 s^{2}-3 d^{10} 4 d$ & $R_{d}^{2}(4 s 4 s, 3 d 4 d)$ & -1.529 & fix & - \\
\hline \multirow{3}{*}{$3 d^{9} 4 s^{2}-3 d^{9} 4 s 5 s$} & $R_{d}^{0}(3 d 4 s, 3 d 5 s)$ & 0.235 & fix & - \\
\hline & $R_{e}^{2}(3 d 4 s, 3 d 5 s)$ & 2.341 & fix & - \\
\hline & $R_{d}^{0}(4 s 4 s, 4 s 5 s)$ & 2.189 & fix & - \\
\hline $3 d^{9} 4 s^{2}-3 d^{9} 4 p^{2}$ & $R_{d}^{1}(4 s 4 s, 4 p 4 p)$ & 36.021 & fix & - \\
\hline $3 d^{9} 4 s^{2}-3 d^{9} 4 p 5 p$ & $R_{d}^{1}(4 s 4 s, 4 p 5 p)$ & 9.939 & fix & - \\
\hline \multirow[t]{2}{*}{$3 d^{10} 4 d-3 d^{9} 4 s 5 s$} & $R_{d}^{2}(3 d 4 d, 4 s 5 s)$ & 0.861 & fix & - \\
\hline & $R_{e}^{2}(3 d 4 d, 4 s 5 s)$ & -0.215 & fix & - \\
\hline \multirow[t]{2}{*}{$3 d^{10} 4 d-3 d^{9} 4 p^{2}$} & $R_{d}^{1}(3 d 4 d, 4 p 4 p)$ & -2.441 & fix & - \\
\hline & $R_{d}^{3}(3 d 4 d, 4 p 4 p)$ & -1.000 & fix & - \\
\hline \multirow[t]{4}{*}{$3 d^{10} 4 d-3 d^{9} 4 p 5 p$} & $R_{d}^{1}(3 d 4 d, 4 p 5 p)$ & -1.156 & fix & - \\
\hline & $R_{d}^{3}(3 d 4 d, 4 p 5 p)$ & -0.018 & fix & - \\
\hline & $R_{e}^{1}(3 d 4 d, 4 p 5 p)$ & -0.675 & fix & - \\
\hline & $R_{e}^{3}(3 d 4 d, 4 p 5 p)$ & -0.276 & fix & - \\
\hline $3 d^{9} 4 s 5 s-3 d^{9} 4 p^{2}$ & $R_{d}^{1}(4 s 5 s, 4 p 4 p)$ & -2.222 & fix & - \\
\hline \multirow[t]{2}{*}{$3 d^{9} 4 s 5 s-3 d^{9} 4 p 5 p$} & $R_{d}^{1}(4 s 5 s, 4 p 5 p)$ & 8.095 & fix & - \\
\hline & $R_{e}^{1}(4 s 5 s, 4 p 5 p)$ & 0.815 & fix & - \\
\hline \multirow[t]{3}{*}{$3 d^{10} 4 p-3 d^{9} 4 s 4 p$} & $R_{d}^{2}(3 d 3 d, 3 d 4 s)$ & -3.715 & fix & - \\
\hline & $R_{d}^{2}(3 d 4 p, 4 s 4 p)$ & -8.208 & fix & - \\
\hline & $R_{e}^{2}(3 d 4 p, 4 s 4 p)$ & -8.398 & fix & - \\
\hline \multirow[t]{2}{*}{$3 d^{9} 4 s 4 p-3 d^{10} 5 p$} & $R_{d}^{2}(4 s 4 p, 3 d 5 p)$ & -3.324 & fix & - \\
\hline & $R_{e}^{1}(4 s 4 p, 3 d 5 p)$ & -3.553 & fix & - \\
\hline
\end{tabular}

Table 2: Transition probabilities, oscillator strengths and energy levels for the copper lines

\begin{tabular}{|c|c|c|c|c|c|c|c|c|c|}
\hline \multirow[t]{2}{*}{ SPECTRUM } & \multicolumn{2}{|c|}{ WAVELENGTHS(nm) } & \multicolumn{2}{|c|}{ LEVELS $\left(\mathrm{cm}^{-1}\right)$} & \multirow{2}{*}{\multicolumn{2}{|c|}{ TRANSITION }} & \multirow{2}{*}{$\begin{array}{c}\mathrm{gA}^{a} \\
10^{8} / \mathrm{sec}\end{array}$} & \multirow[t]{2}{*}{$\overline{\mathrm{gf}^{b}}$} & \multirow{2}{*}{$\begin{array}{c}\mathrm{gA}^{b} \\
10^{8} / \mathrm{sec}\end{array}$} \\
\hline & $\mathrm{Ar}$ & Vac & Lower & Upper(eV) & & & & & \\
\hline $\mathrm{Cu} \mathrm{I}$ & 510.55 & 510.69 & 11202.6 & - $30783.7(2.65)$ & $4 s^{2}{ }^{2} D_{5 / 2}$ & $-\quad 4 p^{2} P_{3 / 2}$ & 0.051 & 0.0197 & 0.0503 \\
\hline $\mathrm{Cu} \mathrm{I}$ & 515.32 & 515.46 & 30535.3 & - $49935.2(4.30)$ & $4 p^{2} P_{1 / 2}$ & $4 d^{2} D_{3 / 2}$ & 4.7 & 1.8710 & 4.6971 \\
\hline $\mathrm{Cu} \mathrm{I}$ & 521.82 & 521.96 & 30783.7 & - $49942.1(4.30)$ & $4 p^{2} P_{3 / 2}$ & $4 d^{2} D_{5 / 2}$ & 5.8 & 2.3417 & 5.7331 \\
\hline $\mathrm{Cu} \mathrm{I}$ & 529.25 & 529.39 & 43514.0 & - $62403.3(5.38)$ & $4 s 4 p{ }^{4} D_{7 / 2}$ & $4 s 5 s{ }^{4} D_{7 / 2}$ & 3.2 & 1.3955 & 3.3215 \\
\hline $\mathrm{Cu} \mathrm{I}$ & 570.02 & 570.18 & 13245.4 & $-\quad 30783.7(2.65)$ & $4 s^{2}{ }^{2} D_{3 / 2}$ & $4 p^{2} P_{3 / 2}$ & 0.014 & 0.0089 & 0.0183 \\
\hline
\end{tabular}

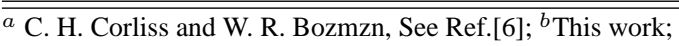

